\title{
CORRIGENDUM
}

\section{Direct seawater desalination by ion concentration polarization}

Sung Jae Kim, Sung Hee Ko, Kwan Hyoung Kang and Jongyoon Han

Nature Nanotechnology 5, 297-301 (2010); published online 21 March 2010; corrected after print 3 July 2013.

In this Article, we measured the electrical current necessary to run the desalination process (Supplementary Information Fig. 3) and reported that the power efficiency of the method was around $3.5 \mathrm{Wh} \mathrm{l}^{-1}$. We have subsequently been unable to reproduce this power efficiency and now believe that our electrical current measurements were wrong. The efficiency of our device is likely to be significantly worse than originally stated. This is because the device has long channels running from the reservoirs to the ion concentration polarization (ICP) zone, and although only a minimal amount of power is required to move ions around the ICP zone, these channels consume a considerable amount of power. Therefore, Supplementary Information Fig. 3, the quoted power efficiency of the device and our discussion surrounding this value in the original paper are not valid. This error does not affect the other results in the Article, and in particular our conclusion that ICP can be used to desalinate sea water. 